() Н.Н. Кушнаренко, М.Ю. Мишко*, Т.А. Медведева, К.Е. Кушнаренко

Читинская государственная медицинская академия, Чита, Россия

Ожирение является одной из наиболее важных медико-социальных проблем современной медицины. В настоящее время все большее внимание уделяется вопросам изучения метаболической активности жировой ткани - источника биологически активных медиаторов, участвующих в процессах сосудистого ремоделирования, иммунологических и воспалительных реакциях. Сегодня ожирение рассматривается не только как независимый фактор риска развития хронических неинфекционных заболеваний (таких как артериальная гипертензия, сахарный диабет 2 типа, патология сердечно-сосудистой системы, злокачественные новообразования), но и в качестве значимого предиктора обменных нарушений (дислипидемии, инсулинорезистентности, гиперурикемии), способствующих раннему формированию метаболического синдрома и подагры. Несмотря на столь очевидную проблему, современное состояние консервативной терапии избыточного веса и ожирения остается неудовлетворительным. У значительного количества пациентов даже после успешного лечения наблюдается рецидив заболевания с восстановлением исходных антропометрических показателей или даже увеличение массы тела. В этой связи в настоящее время более широко стали применять хирургические методы коррекции ожирения. Однако наряду с ожидаемыми положительными эффектами лечения бариатрические операции могут сопровождаться развитием ряда специфических хирургических осложнений и нарушением энергетического метаболизма с усугублением существующей полиморбидной патологии. Данное клиническое наблюдение демонстрирует ассоциацию ожирения с тяжелыми нарушениями метаболизма, реализующими свое патологическое влияние в виде развития у пациента хронической тофусной подагры в 2-летний период после проведенного лапароскопического бандажирования желудка, и позволяет наглядно оценить возможные причины дебюта заболевания, динамику состояния пациента, характер и особенности течения суставного синдрома в послеоперационном периоде.

КЛЮЧЕВЫЕ СЛОВА: ожирение; метаболический синдром; гиперурикемия; подагра; бариатрическая хирургия; клинический случай.

\title{
A CASE OF CHRONIC TOPHI GOUT IN MORBIDLY OBESE PATIENT AFTER LAPAROSCOPIC ADJUSTABLE GASTRIC BANDING
}

(c) Natalia N. Kushnarenko, Marina Yu. Mishko*, Tatyana A. Medvedeva, Kirill E. Kushnarenko

Chita State Medical Academy, Chita, Russia

Currently, increasing attention is being paid to studying the metabolic activity of adipose tissue, a source of biologically active mediators involved in vascular remodeling, immunological and inflammatory reactions. Today obesity is considered not only as independent risk factor of development of chronic noninfectious diseases (such as arterial hypertension, diabetes mellitus types 2, pathology of a cardiovascular system, malignant neoplasms), but also as a significant predictor of metabolic disorders (dyslipidemia, insulin resistance, hyperuricemia), contributes to the early formation of metabolic syndrome and goat. Despite such an obvious problem, the current state of conservative therapy for overweight and obesity remains unsatisfactory. Most of patients, even after successful treatment, have a recurrence of the disease with the restoration of the initial anthropometric indicators or even an increase in body weight. In this regard, currently more widely used surgical methods of correction of obesity. However along with the expected positive effects of treatment bariatric surgery may be accompanied by the development of a number of specific surgical complications and impaired energy metabolism with aggravation of the existing polymorbid pathology. This clinical case demonstrates the association of obesity with the development of metabolic disorders, illustrates the development of severe tophaceous gout in a patient during 2 years after laparoscopic gastric banding. It shows the possible reasons for its debut and reflects the dynamics of the patient's condition, as well as the nature and characteristics of the course of the joint syndrome in the postoperative period.

KEYWORDS: obesity; metabolic syndrome; hyperuricemia; gout; bariatric surgery; case report. 


\section{АКТУАЛЬНОСТЬ}

В настоящее время ожирение признано «глобальной неинфекционной эпидемией» ввиду высокой распространенности. По прогнозам ВОЗ, к 2025 г. почти половина населения планеты будет иметь избыточный вес и ожирение [1].

Установлено, что ожирение является важнейшим фактором риска возникновения и прогрессирования множественной полиорганной патологии - дислипидемии, атеросклероза, артериальной гипертензии (АГ), сахарного диабета (СД), заболеваний опорно-двигательного аппарата [2]. Накоплено достаточно данных о связи ожирения и метаболического синдрома (MC) с гиперурикемией (ГУ) и подагрой [3, 4].

Однако при использовании традиционных немедикаментозных и медикаментозных методов лечения ожирения достичь желаемых результатов удается не всегда. По данным Национального института здоровья США, 30-60\% пациентов, похудевших с помощью диеты и физической нагрузки, через год возвращаются к исходной массе тела, а через 5 лет практически все больные восстанавливают или даже превышают исходные параметры [5]. К тому же бесконтрольное соблюдение низкокалорийной диеты небезопасно ввиду увеличения риска смертности от нарушений сердечного ритма и лактатацидоза. Долгосрочная эффективность медикаментозной терапии ожирения и МС изучена недостаточно. В метаанализе (Padwal R. и соавт.) среднее снижение массы тела за год при лечении орлистатом составило 2,3-3,1 кг, сибутрамином - 34,9 кг [6]. Также нельзя забывать и о безопасности препаратов: в исследовании SCOUT длительный прием сибутрамина у пожилых пациентов, страдающих ожирением, с уже существующими сердечно-сосудистыми заболеваниями привел к увеличению риска развития инфаркта миокарда и инсульта в среднем на 16\%, что явилось причиной прекращения терапии [7].

В связи с этим ведется активный поиск новых высокоэффективных методов лечения, среди которых ведущая роль принадлежит бариатрической хирургии. К 2000 г. в США выполнено около 1 млн таких операций, составляющих более 200 тыс. в год. В Европе на сегодняшний день проводится около 200 тыс. операций в год, в России - чуть более 2000, и частота их продолжает расти.

В большей степени эффективность снижения веса после бариатрических вмешательств зависит от вида выполненной операции. В среднем эффект операций проявляется в течение 1-1,5 лет, а процент потери избыточной массы тела составляет от $45 \%$ до $95 \%$. В ряде исследований показано, что в результате положительных метаболических эффектов, как связанных с потерей веса, так и независимых от него, после бариатрических операций (БО) наблюдается значительное снижение ассоциированных с MC показателей, таких как артериальное давление (АД), уровень глюкозы крови, инсулина, триглицеридов, холестерина, мочевой кислоты (МК) сыворотки крови и общей смертности $[8,9]$.

Однако, несмотря на все положительные стороны бариатрической хирургии, далеко не всегда коррекция веса приводит к нормализации метаболических нарушений. Одной из возможных причин является ряд осложнений, которые сопутствуют этим методам лечения.
Наиболее изученными являются осложнения, связанные с последствиями оказания хирургического пособия: соскальзывание манжеты при бандажировании и развитие пролежня желудка, стеноз выходного отдела и перитонит, демпинг-синдром, дилатация пищевода, несостоятельность анастомоза, развитие эрозий и язв анастомоза, частым осложнением шунтирующих операций является синдром мальабсорбции. К тому же, бариатрическая операция не всегда является гарантией нормализации веса. Немаловажная роль в данном случае принадлежит тщательному отбору пациентов, являющихся кандидатами на бариатрическое вмешательство и активному наблюдению пациентов в послеоперационном периоде. У ряда пациентов могут возникать нарушения метаболических процессов в виде рецидива ожирения в отдаленный период, усугубления ГУ, развития и прогрессирования подагры. Установлено, что несмотря на снижение индекса массы тела (ИМТ) после бандажирования желудка, достигающее максимальных значений к первому году наблюдения $\left(34,6 \pm 5,5 \mathrm{kr} / \mathrm{M}^{2}\right)$, на 3-й и 5-й годы ИМТ вновь восстанавливается, хоть и несколько меньше исходных значений [10].

С этих позиций демонстрация пациента с морбидным ожирением после проведенного лапароскопического регулируемого бандажирования желудка (ЛРБЖ) отражает ассоциацию ожирения с развитием метаболических нарушений и усугубление этих нарушений в послеоперационном периоде.

\section{ОПИСАНИЕ СЛУЧАЯ}

Пациент К., 35 лет, специалист госслужбы, ведет малоподвижный образ жизни, вредных привычек не имеет. Отягощена наследственность: мать пациента страдает ожирением, АГ, СД 2 типа.

Анамнез заболевания. С детства страдал ожирением. В возрасте 17 лет ИМТ составлял 39 кг/м². В 28 лет ИМТ увеличился до 46 кг/м², диагностирована АГ с максимальным уровнем АД до 180/110 мм рт.ст. Традиционные немедикаментозные (низкокалорийная диета) и медикаментозные методы лечения (сибутрамин 10 мг) были неэффективны. Ожирение прогрессировало, ИМТ к 30-летнему возрасту достиг 48 кг/м². В 2012 г. пациенту было проведено ЛРБЖ. Потеря массы тела в течение 8 мес составила 30 кг, снижение ИМТ до 32,5 кг/м². Пациенту рекомендовано продолжить соблюдение диеты, сохранение прежнего режима физической активности. В течение 2 лет после проведения ЛРБЖ наблюдался амбулаторно. На фоне приема ингибиторов ангиотензинпревращающего фермента (лизиноприл 10 мг) сохранялись целевые уровни АД. Периодически проводился контроль общеклинических и биохимических показателей, согласно амбулаторной документации, однократно наблюдалось повышение уровня МК сыворотки крови до 574 мкмоль/л.

Приступ острого подагрического артрита, внезапно возникший в ночные часы и характеризующийся интенсивными ноющими болями «сверлящего» характера, отеком и гиперемией в области первых плюсне-фаланговых суставов обеих стоп, усиливающихся в ночное время, впервые появился спустя 2 года после ЛРБЖ. Пациент обратился к участковому терапевту, при физикальном обследовании выявлены отек и гиперемия первых плюс- 
не-фаланговых суставов стоп, отек голеностопных, локтевых суставов.

Данные амбулаторного обследования: в общем анализе крови выявлено повышение СОЭ до 45 мм/ч; в биохимическом анализе крови (в скобках указана норма): повышение уровня МК до 725,5 мкмоль/л (154,7-428,4), С-реактивного белка до 121,1 мг/л (0-5), уровень холестерина (XС) - 6,26 ммоль/л (3,3-5,5), ХС липопротеидов высокой плотности (ЛПВП) - 1,34 ммоль/л $(0,9-1,9)$, ХС ЛПНП - 3,34 ммоль/л (1,5-3,3), триглицеридов 3,24 ммоль/л (0,14-1,70); в общем анализе мочи выявлена протеинурия до 0,033 г/л и цилиндрурия (гиалиновые 1-1-2 в п.зр.). Уровень МК в сыворотке крови за время наблюдения максимально достигал 825 мкмоль/л, в синовиальной жидкости при проведении поляризационной микроскопии были обнаружены кристаллы моноурата натрия (рис. 1). Больному диагностирован острый подагрический артрит, купировавшийся назначением нестероидных противовоспалительных средств (НПВС). На фоне базисной терапии Аллопуринолом в дозе 200 мг в сутки в течение 3-месячного периода целевые значения уровня МK не были достигнуты, появилась непереносимость препарата в виде диспепсического синдрома (тошнота, рвота, стеаторея), прием Аллопуринола прекратил. Суставной синдром непрерывно рецидивировал, обострения наблюдались 1-2 раза в месяц с вовлечением в процесс голеностопных, коленных, локтевых, плечевых суставов, суставов кистей. Эпизоды острого артрита купировал приемом Нимесулида. Спустя год от дебюта суставного синдрома отмечалось быстрое формирование множественных тофусов. Локализация тофусов была различной: над областью локтевых, голеностопных суставов, ушных раковин. Наблюдалось быстрое увеличение количества и размеров тофусов, что привело к нарушению функции суставов. В сентябре 2015 г. проведено оперативное удаление тофусов над областью локтевых суставов. Пациент наблюдался амбулаторно с диагнозом: основной: Вторичная подагра, метаболический вариант, тофусная форма, хроническое интермиттирующее течение, с поражением I плюснефаланговых суставов стоп, голеностопных, коленных, локтевых суставов. Оперативное удаление тофусов левого локтевого сустава в 2015 г. Хроническая уратная нефро-

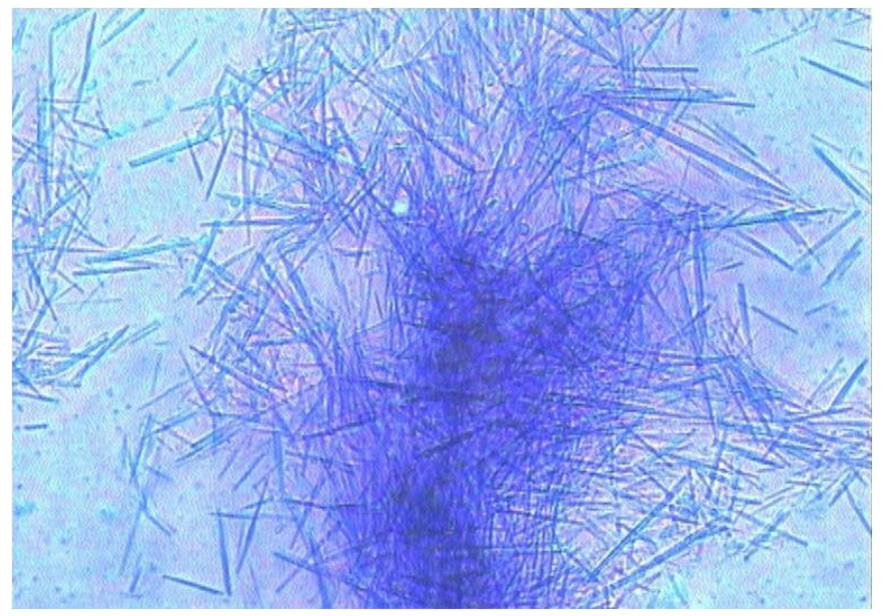

Рисунок 1. Поляризационная микроскопия: кристаллы моноурата натрия характерной игловидной формы, обнаруженные в синовиальной жидкости патия. ХБП I (СКФ-98 мл/мин. CKD-EPI). Нарушение функции суставов I степени. Фоновый: Состояние после бандажирования желудка от 2012 г. (ожирение с ИМТ 48 кг/м²). Алиментарно-конституциональное ожирение 3 степени (ИМТ 48 кг/м², на фоне терапии достигнута избыточная масса с ИМТ 27 кг/м²). Метаболически нездоровый фенотип. Высокий риск кардиометаболических осложнений по шкале CMDS. Неалкогольная жировая болезнь печени, минимальной степени активности, стадия неуточненных фиброзных изменений. Дислипидемия. Conymcmsyющий: Гипертоническая болезнь ІІ стадии, 2 степени, риск 3. Риск по Framingham 1\%.

Ухудшение состояния в июле 2017 г., когда внезапно отмечает появление болей в животе, рвоты съеденной пищей и выпитой жидкостью после каждого приема пищи, геморрагических высыпаний на коже. 18 августа 2017 г. в экстренном порядке был госпитализирован в отделение хирургии Дорожной клинической больницы. При обследовании диагностирована прикрытая перфорация желудка с формированием перигастрального абсцесса и перитонита вследствие развития пролежня в зоне бандажа, проведены операция удаления бандажа, ушивание перфоративного отверстия, санация и дренирование брюшной полости. Послеоперационный период протекал тяжело, с развитием левосторонней полисегментарной пневмонии, тяжелой анемии, эпизода острой почечной недостаточности с повышением уровня креатинина сыворотки крови до 285 мкмоль/л, похудением больного, выраженными метаболическими нарушениями. В раннем послеоперационном периоде у пациента рецидивировал острый приступ подагрического артрита с вовлечением в процесс I плюснефаланговых суставов стоп, голеностопных, лучезапястных и локтевых суставов с выраженными воспалительными проявлениями и нарушением функции суставов, лабораторно в о/а крови наблюдалось повышение СОЭ до 48 мм/ч, в биохимическом анализе крови - гиперурикемия до 851 мкмоль/л и повышение уровня С-реактивного белка до 148 мг/л. В связи с невозможностью назначения НПВС и колхицина в ранний послеоперационный период, для уменьшения интенсивности суставного синдрома пациенту рекомендованы НПВС местно на область пораженных суставов, физиолечение (электрофорез с новокаином).

Результаты дальнейшего наблюдения. После выписки пациент находился под наблюдением в амбулаторных условиях, отмечалось рецидивирующее течение подагры с вовлечением в процесс крупных (голеностопных, коленных, локтевых) и мелких суставов стоп и кистей, с затяжными приступами артрита в ночное время, отличающиеся недостаточным эффектом на прием НПВС. Спустя 6 мес после ЛРБЖ наблюдалось увеличение веса до 115 кг, вновь начали формироваться тофусы над областью локтевых суставов, коже левой ушной раковины, сохранялись высокие уровни МК сыворотки крови (876, 783 и 659 мкмоль/л через 1, 3 и 6 мес наблюдения соответственно). На фоне строгой диеты и базисной терапии Фебуксостатом 80 мг 1 раз в сутки удалось достигнуть снижения ГУ до 520 мкмоль/л. В настоящее время пациент продолжает соблюдать гипопуриновую диету, обострения подагры возникают до 2 раз в год, без четкой связи с провоцирующими факторами, купируются Колхицином 0,0005 мг в сутки. 


\section{ОБСУЖДЕНИЕ}

Все развивающиеся после хирургического лечения ожирения метаболические сдвиги принято подразделять на связанные с потерей избыточного веса, независимые от редукции массы тела и комбинированные положительные эффекты. Причем выраженность метаболических эффектов напрямую зависит от вида операции.

Henry Buchwald и соавт. (2011) в проведенном метаанализе установили, что средняя величина потери массы тела у больных после всех БО составила 61,2\%, в том числе у пациентов после ЛРБЖ - 47,5\%, после желудочного шунтирования - 61,6\%, гастропликации - 68,2\%, после билиопанкреатического шунтирования - 70,1\%. Наряду с потерей массы тела произошли нормализация показателей углеводного, липидного обменов, достижение целевых уровней АД у 75\% пациентов [11].

Нами проанализирован опыт применения и влияния такого рода БО на динамику метаболических сдвигов в отдаленные сроки оперативного лечения. Фишман и соавт. представили данные 3-летнего наблюдения пациентов после ЛРБЖ [10]. У 43\% пациентов наблюдалась нормализация АД, у 59\% - улучшение показателей липидного обмена, ремиссия сахарного диабета 2 типа у 47\% больных. Однако в более отдаленные сроки после установки желудочного бандажа положительные метаболические эффекты операции уменьшались, вновь происходило увеличение массы тела. Авторы связывают такой неблагоприятный исход с сохранением естественного пассажа пищи по всем отделам желудочно-кишечного тракта и отсутствием каких-либо изменений продукции гормонов, регулирующих массу тела и пищевое поведение.

В настоящее время целесообразным является выделение групп пациентов с «метаболически здоровым фенотипом ожирения» (МЗФ) и «метаболически нездоровым фенотипом» (МНЗФ) [12]. Так, при сравнении эффективности операции бандажирования желудка в группах больных с МНЗФ и с МЗФ было показано, что использовать данное вмешательство с целью коррекции МС нецелесообразно ввиду недостаточного эффекта. В большей степени это связано с отсутствием синдрома кишечной мальабсорбции, который наблюдается при других видах БО, в частности, при комбинированных пособиях [10]. Также после ЛРБЖ сохранен контакт пищи с грелин-продуцирующей зоной желудка, в связи с этим долгое время отмечается увеличение уровня грелина натощак, которое индуцируется снижением калорийности и объема поступающей пищи. Это вызывает стимуляцию центра голода в гипоталамусе, повышение аппетита, что приводит к увеличению массы тела и утрате эффекта от проведенного бандажирования желудка $[13,14]$. К тому же при операциях ЛРБЖ часто развиваются осложнения, связанные с самой системой бандажа, - смещение манжеты, стеноз выходного отдела и развитие пролежня желудка (12,6\% случаев), расширение пищевода и эрозивное поражение желудочно-кишечного тракта [10].

Важнейшим аспектом метаболических расстройств является изменение уровня МК сыворотки крови и связанное с ГУ развитие подагры [4]. Бариатрические хи- рургические вмешательства приводят к ускорению катаболических процессов в ассоциации с длительными периодами голодания и дегидратацией. Высокобелковая диета в послеоперационном периоде также может способствовать развитию ГУ и подагры. В настоящее время в литературе имеются неоднозначные данные о взаимосвязи заболеваемости подагрой и частоты атак подагрического артрита у пациентов, подвергшихся бариатрическим вмешательствам на желудке $[15,16,17]$.

В мультицентрическом проспективном исследовании Swedish Obese Subjects Study показано, что у пациентов с ожирением, подвергшихся БО на желудке, отмечались значительная потеря веса, уменьшение уровня МК сыворотки крови и снижение частоты обострений подагрического артрита по сравнению с контрольной группой [15]. Снижение и нормализация уровня МК сыворотки крови наблюдались в течение года после оперативного лечения и сохранялись на протяжении 20 лет. Кроме того, результаты исследования показали, что БО предотвращают развитие ГУ у пациентов с исходно нормальными уровнями МК сыворотки крови. Так, было установлено, что после бариатрических вмешательств наблюдалась более низкая заболеваемость подагрой (138 случаев в основной группе против 201 у респондентов контрольной группы за период наблюдения с 1987 по 2001 гг.) [15].

Ряд других авторов в своих исследованиях показали, что частота ранних послеоперационных приступов подагры после БО значительно выше, чем в группе пациентов, подвергшихся другим хирургическим вмешательствам. Однако частота атак значительно уменьшается в период от 1 мес до года после операции [16].

Dalbeth и соавт. (2014) в своих работах доказали, что бариатрические вмешательства приводят к снижению системной воспалительной реакции на кристаллы моноурата натрия, что также может частично способствовать снижению частоты развития и рецидивов подагры в группе пациентов, перенесших хирургическое вмешательство [9].

Үео С. и соавт. (2019) проведено обобщение имеющихся данных о взаимосвязи бариатрических вмешательств, ГУ и подагры. Проанализировано 20 исследований с общим числом пациентов 5233. Средний предоперационный ИМТ у пациентов составлял 45,2 кг/м², средний предоперационный уровень МК сыворотки крови - 574,6 мкмоль/л. В первый послеоперационный месяц отмечался рост уровня МК сыворотки крови. Однако дальнейший анализ показал снижение уровней МК с третьего послеоперационного месяца и далее, длившееся до 3 лет [17]. В ряде работ показано, что приступы подагры могут развиваться и в раннем послеоперационном периоде, в среднем течение 8 дней после операции. Так, Kang и соавт. (2008) обнаружили, что более частые рецидивы подагры были характерны для пациентов с высоким предоперационным уровнем МК сыворотки крови и предшествующим в предоперационном периоде лечением колхицином [18].

И только немногочисленные исследования свидетельствуют, что БО являются фактором повышенного риска обострений подагрического артрита, а снижение веса не приводит к снижению активности подагры [19]. 
Однако прогрессирование подагры и ГУ может наблюдаться не только после проведения БО, но и при любом другом значительном снижении веса посредством медикаментозных воздействий $[20,21]$. С другой стороны, Nguyen и соавт. (2017) в исследованиях показали, что постепенное снижение ИМТ достоверно уменьшало риск рецидива подагрического артрита [22].

В нашем случае, напротив, несмотря на проведенное хирургическое лечение ожирения, наблюдался клинический дебют подагры после операции, а также усугубление течения суставного синдрома в момент развития слиппадж-синдрома и в послеоперационном периоде.

Пациенту К. было выполнено бандажирование желудка. В течение первого года после ЛРБЖ потеря веса составила $42 \%$, через 2 года - 58\% от исходной массы тела. Клинически отмечал улучшение самочувствия, уменьшение одышки и повышение толерантности к физической нагрузке, были достигнуты целевые показатели АД. Но, несмотря на это, спустя 2 года после проведения вмешательства у пациента дебютировал острый подагрический артрит с вовлечением большого количества суставов, формированием множественных тофусов, характеризующийся быстропрогрессирующим течением с нарушением функции верхних конечностей, что в дальнейшем потребовало оперативного удаления тофусов. Болевой синдром непрерывно рецидивировал, требуя постоянного приема анальгетиков, целевые уровни МК достигнуты не были.

Мы попытались проанализировать причинно-следственные механизмы развития подагры у нашего больного. Во-первых - длительно текущее осложненное ожирение. Пациент длительно страдал ожирением, в течение 22 лет ИМТ пациента в среднем составлял 42 кг/м². В настоящее время придается большое значение метаболической и гормональной активности жировой ткани [23]. Продолжается изучение роли инсулинорезистентности в развитии подагры. Длительно текущее прогрессирующее ожирение у молодого мужчины индуцировало изменение гормональной активности, секрецию большого количества биологически активных веществ, обладающих локальными и системными метаболическими эффектами, регулирующих гомеостаз всего организма. К таким соединениям относят цитокины, фактор некроза опухолей альфа, лептин, резистин, ростовые факторы, приводящие к развитию инсулинорезистентности и лептинорезистентности. В свою очередь, инсулин, действуя в проксимальных канальцах, стимулирует реабсорбцию моноуратов натрия, что является одной из причин ГУ и подагры [24].

Неоднозначны механизмы взаимодействия ГУ и АГ. Мы наблюдали ранний дебют АГ у нашего пациента в возрасте 28 лет. По данным многочисленных исследований установлено, что у пациентов с избыточной массой тела АГ встречается в 2,9 раза чаще, чем у лиц с нормальной массой тела [25]. Наличие АГ сопровождается развитием гломерулярной ишемии, гиперфильтрации и нефроангиосклероза, вызывая нарушение уратного метаболизма, развитие ГУ и подагры [26]. С другой стороны, наличие подагры повышает риск возникновения и прогрессирования АГ, что может быть связано с развитием эндотелиальной дисфункции и гломеруло- тубулярных повреждений, индуцирующих активизацию ренин-ангиотензин-альдостероновой системы и повышение АД на фоне ГУ [27]. Наличие же МС при подагре способствует развитию АГ, поскольку патогенетические механизмы АГ напрямую ассоциированы с инсулинорезистентностью, ожирением и нарушениями углеводного обмена - компонентами МС [24]. На настоящий момент АГ у пациента К. требует контроля, проводится коррекция антигипертензивной терапии.

Также в ряде работ сообщается о послеоперационном повышении уровня МК в сыворотке крови по причине почечной дисфункции, вызванной операцией, катаболическими сдвигами и резким снижением массы тела $[9,28]$. С другой стороны, российскими исследователями показано, что быстрое снижение массы тела (более 15\% от исходной в течение 6 мес) усугубляет поражение тубулярного эпителия и развитие почечной дисфункции [29]. Все это в конечном итоге может привести к дебюту или рецидиву подагры.

Немаловажным является и тот факт, что на этапе отбора и подготовки пациента к оперативному лечению не учитывался ряд факторов, таких как отягощенная наследственность, особенности питания и образа жизни, не были проведены соответствующая диагностика и коррекция имеющихся метаболических нарушений.

\section{ЗАКЛЮЧЕНИЕ}

Продемонстрированный случай отражает взаимосвязь ожирения, ГУ и подагры. Особенностью данной клинической ситуации является дебют подагрического артрита после ЛРБЖ и усугубление его течения в связи с развитием слиппадж-синдрома. Тяжелые метаболические расстройства у нашего пациента, усугубившиеся на фоне гиповолемии и дегидратации, активизация катаболических процессов в связи с развитием перфорации желудка в зоне бандажа могли способствовать обострению и нарастанию проявлений суставного синдрома. Высокобелковая диета в послеоперационном периоде, вероятно, привела к увеличению синтеза и экскреции МК, способствуя тем самым обострению суставного синдрома и прогрессированию заболевания.

\section{ДОПОЛНИТЕЛЬНАЯ ИНФОРМАЦИЯ}

Источник финансирования. Проведение исследования и подготовка статьи осуществлены на личные средства авторского коллектива.

Согласие пациента. Добровольное информированное согласие пациента на публикацию медицинской информации в обезличенной форме в журнале «Ожирение и метаболизм» получено.

Конфликт интересов. Авторы декларируют отсутствие явных и потенциальных конфликтов интересов, связанных с публикацией настоящей статьи.

Информация о вкладе каждого автора.

Кушнаренко Н.Н. - разработка концепции и дизайна, проверка критически важного интеллектуального содержания, окончательное утверждениедля публикации рукописи; Мишко М.Ю.-сбор, анализ и интерпретация данных, написание текста; Медведева Т.А. - сбор, анализ и интерпретация данных, написание текста; Кушнаренко К.Е. - анализ полученных данных, окончательное утверждение для публикации рукописи. Все авторы внесли значимый вклад в проведение исследования и подготовку статьи, прочли и одобрили финальную версию статьи перед публикацией. 


\section{СПИСОК ЛИТЕРАТУРЫ | REFERENCES}

1. Лазнам С.С., Берштейн Л.Л., Гришкин Ю.Н. Значение оценки факторов сердечно-сосудистого риска в прогнозировании ишемической болезни сердца. // Вестник Российской военномедицинской академии. - 2011. — №4. - C. 185-194. [Laznam SS, Berstein LL, Grishkin YN. The role of risk factors assesement in predicting coronary heart disease. Vestnik Rossiiskoi voennomeditsinskoi akademii. 2011;(4):185-194. (In Russ).]

2. Allison DB, Downey M, Atkinson RL, et al. Obesity as a disease: a white paper on evidence and arguments commissioned by the Council of the Obesity Society. Obesity. 2008;16(6):1 161-1177. doi: https://doi.org/10.1038/oby.2008.231

3. Choi HK, Ford ES, Li CY, Curhan G. Revalence of the metabolic syndrome in patients with gout: The Third National Health and Nutrition Examination Survey. Arthritis Rheum. 2007;57(1):109-115. doi: https://doi.org/10.1002/art.22466

4. Насонова В.А., Елисеев М.С., Барскова В.Г. Влияние возраста на частоту и выраженность признаков метаболического синдрома у больных подагрой. // Современная ревматология. — 2007. — №1. - C.31-36. [Nasonova VA, Eliseev MS, Barskova VG. Impact of age on the frequency and magnitude of the signs of metabolic syndrome in patients with gout. Modern Rheumatology Journal. 2007;1 (1):31-36. (In Russ).] doi: https://doi.org/10.14412/1996-7012-2007-436

5. Савельева Л.В. Современная концепция лечения ожирения. // Ожирение и метаболизм. - 2011. — Т. 8. - №1. C. 51-56. [Savel'eva LV. Sovremennaya kontseptsiya lecheniya ozhireniya. Obesity and metabolism. 2011;8(1):51-56. (In Russ).] doi: https://doi.org/10.14341/2071-8713-5191

6. Padwal R, Li SK, Lau DC. Long-term pharmacotherapy for overweight and obesity: a systematic review and meta-analysis of randomized controlled trials. Int J Obes Relat Metab Disord. 2003;27(12):1437-1446. doi: https://doi.org/10.1038/sj.ijo.0802475

7. Karlsson J, Taft C, Rydén A, et al. Ten-year trends in health-related quality of life after surgical and conventional treatment for severe obesity: the SOS intervention study. Int J Obes (Lond). 2007;31(8);1248-1261. doi: https://doi.org/10.1038/sj.ijo.0803573

8. Sjostrom L, Narbro K, Sjostrom CD, et al. Effects of bariatric surgery on mortality in Swedish obese subjects. N Eng/ J Med. 2007;357:741-752. doi: https://doi.org/10.1056/NEJMoa066254

9. Dalbeth N, Chen P, White M, et al. Impact of bariatric surgery on serum urate targets in people with morbid obesity and diabetes: a prospective longitudinal study. Ann Rheum Dis. 2014;73(5):797-802. doi: https://doi.org/10.1136/annrheumdis-2013-203970

10. Фишман М.Б., Седов В.М., Ван Я. Регулируемое бандажирование желудка. Иллюзии и реальность. // Вестник хирургии имени И.И. Грекова. - 2016. T. 175. - №2. - C. 25-29. [Fishman MB, Sedov VM, Yan V. Laparoscopic adjustable gastric banding. Illusions and reality. Vestn Khir Im / / Grek. 2016;175(2):25-29. (In Russ).] doi: https://doi.org/10.24884/0042-4625-2016-175-2-25-29

11. Buchwald H, Danette MO. Metabolic/bariatric surgery worldwide 2011. Obes Surg. 2013;23(4):427-436. doi: https://doi.org/10.1007/s11695-012-0864-0

12. Отт А.В., Чумакова Г.А., Веселовская Н.Г. Эпикардиальное ожирение как один из основных критериев метаболически тучного фенотипа ожирения // Сибирское медицинское обозрение. - 2017. - №4. - C.44-53. [Ott AV, Chumakova GA, Veselovskaya NG. Epicardial obesity as one of the main criteria for the metabolic obese phenotype of obesity. Siberian Medical Review. 2017;(4):44-53. (In Russ).] doi: https://doi.org/10.20333/2500136-2017-4-44-53

13. Бабенко А.Ю., Неймарк А.Е., Анисимова К.А., Гринева Е.Н. Эффекты бариатрических операций на уровень гормонов, регулирующих массу тела. В чем основа успеха? // Ожирение и метаболизм. - 2014. - Т. 11. - №4. - C. 3-11. [Babenko AY, Neymark AE, Anisimova KA, Grineva EN. Effects of bariatric surgery on the level of hormones that regulate body weight. What is the basis of success? Obesity and metabolism. 2014;11(4):3-11. (In Russ).] doi: https://doi.org/10.14341/omet201443-11

14. Морбидное ожирение. / Под ред. Дедова И.И. М.: Медицинское информационное агентство, 2014 [Dedov II, editor. Morbidnoe ozhirenie. Moscow: Meditsinskoe informatsionnoe agentstvo; 2014. (In Russ).]
15. Maglio C, Peltonen M, Neovius M, et al. Effects of bariatric surgery on gout incidence in the Swedish Obese Subjects study: a non-randomised, prospective, controlled intervention trial. Ann Rheum Dis. 2017;76(4):688-693. doi: https://doi.org/10.1136/annrheumdis-2016-209958

16. Romero-Talamás H, Daigle CR, Aminian A, et al. The effect of bariatric surgery on gout: a comparative study. Surg Obes Relat Dis. 2014;10(6):1161-1165. doi: https://doi.org/10.1016/j.soard.2014.02.025

17. Yeo C, Kaushal S, Lim B, et al. Impact of bariatric surgery on serum uric acid levels and the incidence of gout-A meta-analysis. Obes Rev. 2019;20(12):1759-1770. doi: https://doi.org/10.1111/obr.12940

18. Kang EH, Lee EY, Lee YJ, et al. Clinical features and risk factors of postsurgical gout. Ann Rheum Dis. 2008;67(9):1271-1275. doi: https://doi.org/10.1136/ard.2007.078683

19. Antozzi P, Soto F, Arias F, et al. Development of Acute Gouty Attack in the Morbidly Obese Population after Bariatric Surgery. Obes Surg. 2005;15(3):405-407. doi: https://doi.org/10.1381/0960892053576802

20. Raziel A. Gout, obesity and bariatric surgery. $A d v$ Obes Weight Manag Control. 2018;8(4):224-229. doi: https://doi.org/10.15406/aowmc.2018.08.00248

21. Zhang Y, Chaisson CE, McAlindon T, et al. The online casecrossover study is a novel approach to study triggers for recurrent disease flares. J Clin Epidemiol. 2007;60(1):50-55. doi: https://doi.org/10.1016/j.jclinepi.2006.04.006

22. Nguyen UDT, Zhang Y, Louie-Gao Q, et al. The Obesity Paradox in Recurrent Attacks of Gout in Observational Studies: Clarification and Remedy. Arthritis Care Res (Hoboken). 2017;69(4):561-566. doi: https://doi.org/10.1002/acr.22954

23. Дедов И.И., Мельниченко Г.А., Бутрова С.А. Жировая ткань как эндокринный орган. // Ожирение и метаболизм. 2006. - T. 3. — №1. — C. 6-13. [Dedov II, Mel'nichenko GA, Butrova SA. Zhirovaya tkan'kak ehndokrinnyy organ. Obesity and metabolism. 2006;3(1):6-13. (In Russ).] doi: https://doi.org/10.14341/2071-8713-4937

24. Денисов И.С., Елисеев М.С., Барскова В.Г. Исходы подагры. Обзор литературы. Часть II. Коморбидные заболевания, риск развития сердечно-сосудистых катастроф и смерти при подагре. // Проблемы практической ревматологии. — 2013. T. 51. — №6. - C. 703-710. [Denisov IS, Eliseev MS, Barskova VG. Gout outcomes. Literature review. Part II. Comorbid diseases, risk of developing cardiovascular catastrophes and death in gout patients. Rheumatology Science and Practice. 2013;51 (6):703-710. (In Russ).] doi: http://doi.org/10.14412/1995-4484-2013-703-10

25. DeMarco VG, Aroor AR, Sowers JR. The pathophysiology of hypertension in patients with obesity. Nat Rev Endocrinol. 2014;10(6):364-376. doi: https://doi.org/10.1038/nrendo.2014.44

26. Roddy E, Choi HK. Epidemiology of gout. Rheum Dis Clin North Am. 2014;40(2):155-175. doi: https://doi.org/10.1016/j.rdc.2014.01.001

27. Маркелова Е.И., Елисеев М.С., Барскова В.Г. Артериальная гипертензия у больных подагрой: основы патогенеза, клиническое значение, диагностика. // Современная ревматология. - 2012. - Т. 6. — №4. - С. 23-30. [Markelova EI, Eliseyev MS, Barskova VG. Arterial hypertension in gout patients: Pathogenesis bases, clinical value, diagnosis. Sovremennaia revmatologiia. 2012;6(4):23-30. (In Russ).]

28. Maciejewski ML, Arterburn DE, Van Scoyoc L, et al. Bariatric Surgery and Long-term Durability of Weight Loss. JAMA Surg. 2016:151(11):1046-1055. doi: https://doi.rog/10.1001/jamasurg.2016.2317

29. Шулькина С.Г. Метаболические, гормональные и генетические факторы в формировании фенотипа ожирения, ихзначение в развитии почечной дисфункции и эффективность терапевтического воздействия: Дис. ... д-ра мед. наук. Пермь; 2018. [Shul'kina SG. Metabolicheskie, gormonal'nye i geneticheskie faktory v formirovanii fenotipa ozhireniya, ikh znachenie $v$ razvitii pochechnoy disfunktsii i effektivnost' terapevticheskogo vozdeystviya. [dissertation] Perm'; 2018. (In Russ).] 
ИНФОРМАЦИЯ ОБ АВТОРАХ [AUTHORS INFO]:

* Мишко Марина Юрьевна [Marina Yu. Mishko, MD]; адрес: Россия, 672090,

г. Чита, ул. Горького, д. 39A [address: 39A Gorky street, 672090 Chita, Russia]; ORCID: https://orcid.org/0000-0003-3243-2951; eLibrary SPIN: 5374-5324; e-mail:mm_you@mail.ru

Кушнаренко Наталья Николаевна, д.м.н. [Natalia N. Kushnarenko, MD, PhD]; ORCID: https://orcid.org/0000-0002-0350-0698; eLibrary SPIN: 9994-0639; e-mail: natalia.kushnarenko1@gmail.com

Медведева Татьяна Александровна [Tatyana A. Medvedeva, MD]; ORCID: https://orcid.org/0000-0001-8410-5827; eLibrary SPIN: 5043-0194; e-mail: saidi-tma@mail.ru

Кушнаренко Кирилл Евгеньевич [Kirill E. Kushnarenko, MD]; ORCID: https://orcid.org/0000-0001-6112-1963;

eLibrary SPIN: 4455-9949; e-mail: kirillkush@rambler.ru

*Автор, ответственный за переписку / Corresponding author.

\section{ЦИТИРОВАТЬ:}

Кушнаренко Н.Н., Мишко М.Ю., Медведева Т.А., Кушнаренко К.Е. Случай развития хронической тофусной подагры у пациента с морбидным ожирением после лапароскопического регулируемого бандажирования желудка // Ожирение и метаболизм. - 2020. - Т. 17. - №1. - С. 93-99. doi: https://doi.org/10.14341/omet9736

\section{TO CITE THIS ARTICLE:}

Kushnarenko NN, Mishko MY, Medvedeva TA, Kushnarenko KE. A case of chronic tophi gout in morbidly obese patient after laparoscopic adjustable gastric banding. Obesity and metabolism. 2020;17(1):93-99. doi: https://doi.org/10.14341/omet9736 